\title{
New packaging of flow sensors with monolithic Integration of micro-channel on chip
}

S. Billat, K. Kliche, R. Gronmaier, P. Nommensen, J. Auber, F. Hedrich, M. Ashauer, R. Zengerle HSG-IMIT

Wilhelm-Schickard-Str. 10, 78052 Villingen-Schwenningen, GERMANY

\begin{abstract}
We report on the integration of a micro flow channel with a thermal flow sensor chip. Two different technologies were investigated: First the fluid channel is performed at the back side of the standard HSGIMIT flow sensor chip using standard silicon etching process (KOH and RIE). The second technology consists of a bond process between the flow sensor die and a polydimethylsiloxane (PDMS) sheet containing the channel. Both sensor types can be used for detecting flow rates in the range of 0.1 $5.0 \mu \mathrm{l} / \mathrm{min}(\mathrm{H} 2 \mathrm{O})$ and/or pressure differences of 10-600 Pa. Compared to our previous sensor design this leads to reduced packaging costs, increased reproducibility (better than $1 \%$ ) and in particular a higher sensitivity at low flow rates. Finally a concept of an adhesive-free packaging of the sensor is presented.
\end{abstract}

\section{Introduction}

At present, flow sensors are mainly used for air flow determination in automotive applications. However an increasing demand is observed for fluid measurements in smallest flow ranges in chemical analytics, medical diagnostics and biotechnological analysis. These new applications require highly precise measurement and reproducibility, short response time, low power consumption, smallest dimensions, adhesive free packaging and low-cost disposable products [1, 2]. Furthermore miniature flow rate sensors are used in a wide range of applications with length scales varying from flow measurements in oil wells to micro reactors [3]. The flow range to be measured varies over several orders of magnitude. A number of the existing flow sensors are based on measurement of the pressure drop across a narrow flow channel [4-7]. The sensors are based on two separate pressure membranes placed on each side of the flow channel. These sensors are usually used as differential pressure sensor and are not really adequate for flow rate determination. The flow sensor presented here is based on one single membrane sensor similar to [8]. This solution eliminates noise from thermal, electrical and mechanical sources that would affect the two pressure membranes differently. A second possibility to integrate the channel over the sensor is presented by [9], where the silicon die is glued directly on a capillary. This last method presents relative high costs for the adhesive and calibration steps which lead to an expensive sensor. By the integration of chip sized micro-channels (figure 1) with the flow-sensor an increased reproducibility $(<1 \%)$ of the sensor and a measurement range of $0.1-5 \mu \mathrm{l} / \mathrm{min}$ can be attained. However a minimal change of the channel geometry implies a large signal deviation by such small geometrical dimensions. Therefore a very precise alignment of the channel over the active sensor is required as well as very small tolerances for its fabrication. Two different technologies were evaluated to combine a highly precise sensor with a low cost disposable system. Afterwards the chips could be integrated in an adhesive free packaging.

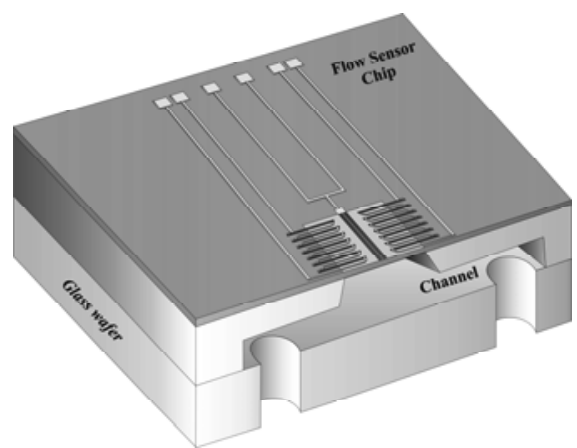

Figure 1:

Schematic cross-section of the flow sensor chip with integrated microfluid-channel at the back side of the die. A glass wafer provides the fluidic connection.

\section{Realisation}

At first a combination of enhanced traditional silicon technologies was used. The channel fabrication is performed by a $\mathrm{KOH}$ etching followed by RIE-etching at the backside of the sensor chip. Finally a full wafer bond with a glass wafer including inlet and outlet drillings for the fluid delivery is performed. 
Figure 2 presents the process flow of the device. The starting material of the sensor fabrication is a (100) n-type silicon double-side polished wafer with a thickness of $300 \mu \mathrm{m}$ and a diameter of $100 \mathrm{~mm}$. After the deposition of the membrane materials (150 nm LPCVD silicon nitride layer on a thin oxide) a $\mathrm{KOH}$ etching of $140 \mu \mathrm{m}$ depth is performed at the back side of the silicon wafer to perform the future cavity under the membrane. The next step is the standard process for the thermopiles fabrication with a $100 \mathrm{~nm}$ n-doped polysilicon layer and a $300 \mathrm{~nm}$ aluminium one. This step needs a photolithography adjustment from back to front side. At this point a light deviation in the adjustment can occur leading to a small change in the sensor sensitivity. Afterwards - in order to protect the thermal sensor against harsh environments - a PECVD $\mathrm{Si}_{3} \mathrm{~N}_{4}$ layer is applied as passivation.

Then the dry etching (RIE) is performed for the channel fabrication. This relative expensive one wafer etching technology is preferred to a simple wafer batch $\mathrm{KOH}$-etching because this step represents the main delicate process.
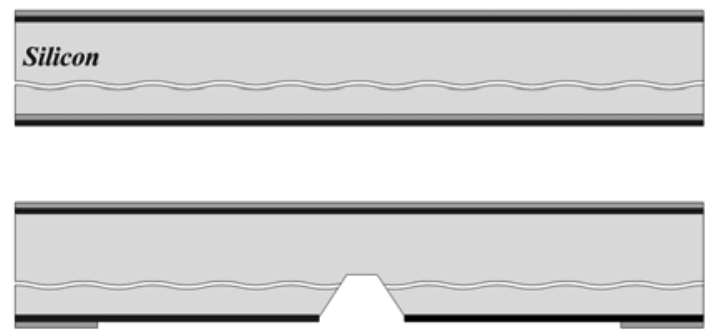

KOH-etching for cavity under the membrane
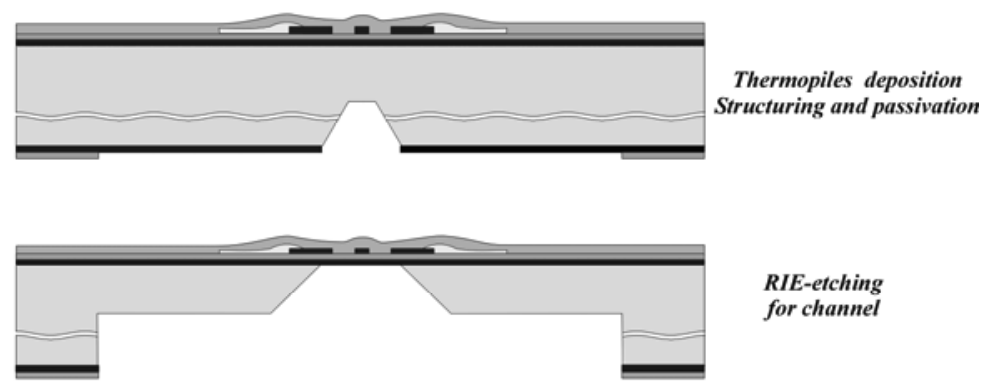

RIE-etching for channel

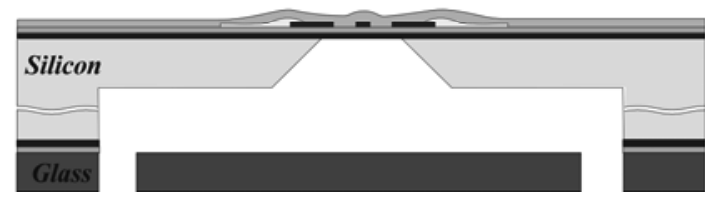

Glass Wafer bond

Figure 2:

Schematic drawing of the process sequence for the chip fabrication. Indeed a too long $\mathrm{KOH}$-etching will rapidly cause a membrane enlargement that consequently would change the heat distribution over the membrane. For example an extension of the membrane from $300 \mu \mathrm{m}$ to $380 \mu \mathrm{m}$ width induces a sensitivity increase of about $40 \%$ in air for a $1 \mathrm{~mm}^{2}$ channel cross section in case of our standard flow sensor [10]. Furthermore free degree of channel design is given by RIE etching which allows the realization of meanders for the fluid channel on an optimized reduced silicon area. With that dry etching the membrane dimensions are well defined and present high fabrication reproducibility.

Finally a full wafer bond with a glass wafer including borings as inlet and outlet provides the fluid delivery. This last step presents the convenience that glass closes the micro-fluid channel and ensures by the way a minimal roughness and a wave less surface. This is definitely a relevant advantage versus injection molding channel fabrication considering the reproducibility of the channel dimensions and thus of the sensor signal and accuracy. As result no personalization calibration during the production process should be necessary. Figure 3 presents the final flow sensor with integrated fluid channel at its backside.

In order to obtain rapidelly more freedom in channel design (e.g. height or rapid realization of meander structures to alter the fluidic resistance), new bonding technologies based on biocompatible materials such as polydimethylsiloxane (PDMS) were developed. Low cost PDMS channel structures were used to define rapidly the influences of different channel geometries on the sensor sensitivity and the measurement range. The used PDMS is a two-component heat-curing system consisting of a base and a curing agent part. After mixing the two components the silicone is dispensed on a dry etched silicon template presenting the negative form of the future channels. After

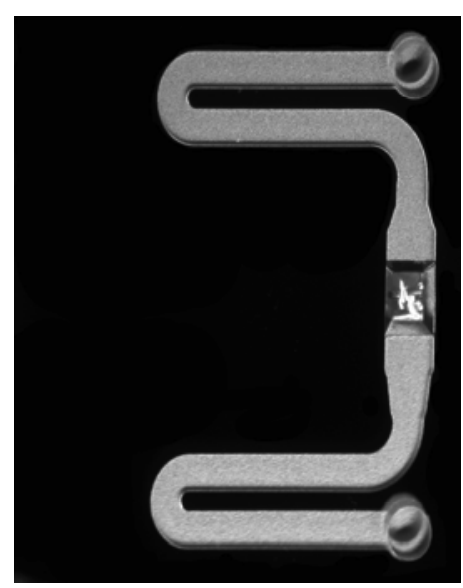

Figure 3:

Picture of backside of the final flow sensor chip with meander fluid channel. spreading and degassing a heat curing at $140{ }^{\circ} \mathrm{C}$ is performed for about $15 \mathrm{~min}$. Consequently, the PDMS structures shrink of almost exactly $3 \%$. Afterwards the PDMS plate containing the channel structures is pealed off the silicon template and stamped in small dies. The following bond process is 
performed after plasma activation of both partners. The process steps are summarised in figure 4 . The bond step is performed chip per chip using a fine placer. Under these conditions the reproducibility of the fabrication and especially the adjustment of the channel over the membrane are not as good as with the alignment obtained by a mask aligner. A failure of about $40 \mu \mathrm{m}$ in adjustment is observed over the channel length, causing an eventual change in the flow status between laminar and turbulent. In the PDMS channel fabrication itself a deviation of the channel height is observed that has several causes. First there is a material shrink during the curing process. Secondly the precision of the dry etching for the silicon template is of about $\pm 2 \mu \mathrm{m}$ which leads to a high failure in percent for the low channel heights which are in our case the focus of the current development. At least a small tolerance is induced by the bond process itself.

\section{Results and discussion}

Flow measurements, sensitivity and measurement range depends on several parameters which highly interact with each other. For example the sensor sensitivity is strongly correlated to the flow resistance and the channel geometry. The increase of the fluid resistance induces a decrease of the flow rate in the sensor and thereby a lower output signal. With a smaller channel height the fluid velocity increases at the same time and the output signal rises. Both phenomena take place simultaneous which means that the sensor sensitivity will follow the most important parameter. Under these conditions an analytical description of the thermal flow sensor would premise a high complexity.

\section{- Standard technologies}

To determine the influence of the tolerances of fabrication technology, a series of air flow measurements were carried out with sensor chips which were picked randomly over a batch. The first investigations were performed with sensors containing straight fluid channel. The fact that flow resistance depends only on the fluid parameters and the channel geometry made this dimension independent from the flow velocity and can be used for a direct comparison of different sensor types like to define the reproducibility of the technology. Figure 5 presents the evolution of the pressure drop over flow for several chips picked over a batch. The pressure drop over the sensor system is a measure for the cross section of the fluid channel and indicates the reproducibility of the

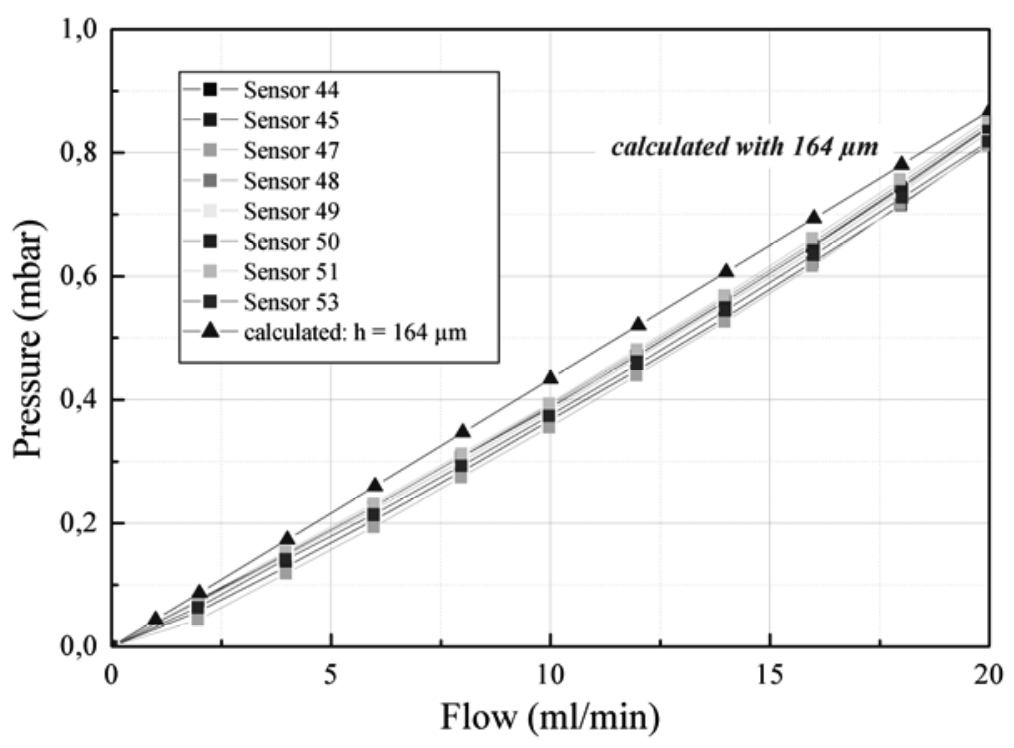

Figure 5:

Air pressure drop over the flow sensor vs. flow rate for several sensors with a $4 \mathrm{~mm}$ channel length. 
flow sensor fabrication. An excellent reproducibility is achieved, since the observed deviations are in the range of the accuracy of the used reference pressure sensor [11].

Using the Hagen-Poisseuille law for a square cross section the flow resistance can be calculated as follows:

$R_{R f}=8 \cdot \varphi \cdot \frac{l \cdot(b+h)^{2}}{(b \cdot h)^{3}} \cdot v \cdot \rho$

Therein $b$ and $h$ represents width and high of a rectangular channel, $\rho$ the fluid density, $v$ the cinematic viscosity and $\varphi$ the coefficient for the cross section form in the flow channel. The RIE etched channel height can be extrapolated from the curve slope assuming a channel width of $820 \mu \mathrm{m}$. In this way the flow sensor presents at its back side a

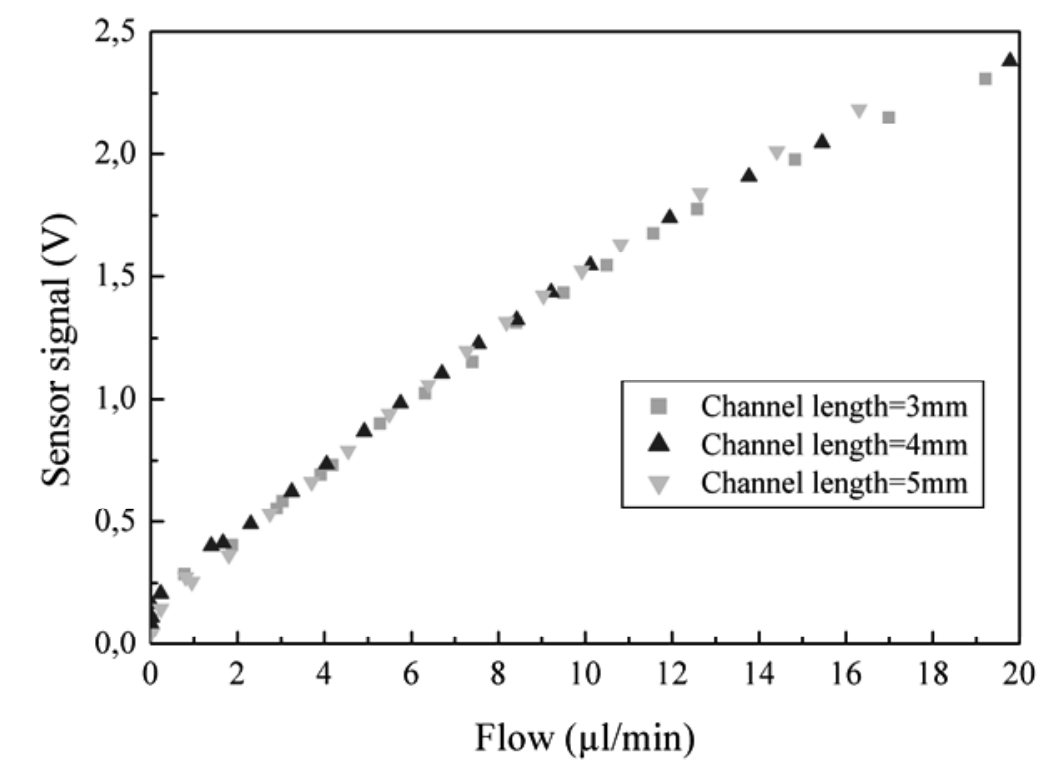

Figure 6:

Output signal of sensors with different channel lengths versus flow. Heat power $=10 \mathrm{~mW}$ and water as fluid

channel height of about $164 \mu \mathrm{m}$. The calculated value fitted very well with the experimental results. Parallel to the channel geometry investigations the flow rate measurements have been carried out to obtain the characteristic curve and show the feasibility of the monolithic integration of micro-channel on sensor chip and thus for flow sensors with different channel lengths. Figure 6 presents the results in a measurement range below $15 \mu \mathrm{l} / \mathrm{min}$. The measurements were performed with water as fluid. The curves present a linear behavior within this low flow measurement range. A perfect overlap of all curves can be pointed out. To conclude a high reproducibility of the sensor signal like accuracy using this etching technology is achieved and improved.

\section{- PDMS technology}

To achieve a better resolution and accuracy of the flow determination in a measurement range below $1 \mu \mathrm{l} / \mathrm{min}$, the sensors with a PDMS layer on top, containing flow channels were characterized.

Figure 7 shows the influence of the channel height on the sensor measurement range and sensitivity, respectively. As expected, there is a significant increase of the sensor signal with decreasing channel heights. For a $2 \mu \mathrm{l} / \mathrm{min}$ reference flow the sensor output signal is about ten times higher for a $25 \mu \mathrm{m}$ channel height than for the one with a height of $200 \mu \mathrm{m}$. For a $25 \mu \mathrm{m}$ high channel a detection limit of $0.05 \mu \mathrm{l} / \mathrm{min}$ is obtained. However, the width of the measurement range decreases considerably with smaller channel heights. The same

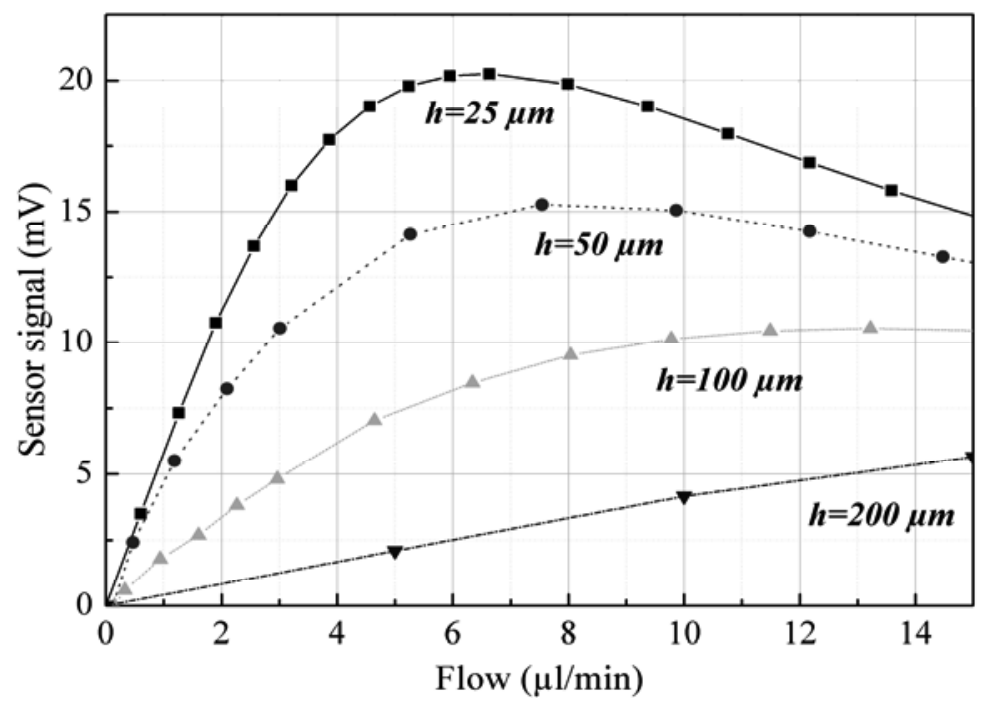

Figure 7:

Sensor signal over flow for different fluid channel heights. Measurements are performed at $10 \mathrm{~mW}$ heating power and water as fluid. measurements are performed in air.

A linear increase of the sensitivity is observed with channel height from $200 \mu \mathrm{m}$ to $50 \mu \mathrm{m}$. By $25 \mu \mathrm{m}$ channel height a lower sensitivity as expected is obtained. One reason for this behavior is the heat dissipation in the upper opposite channel wall. 
Furthermore, one of the drawbacks of PDMS material is its elasticity. For applications with air, under a relative pressure of about 100 mbar and a $25 \mu \mathrm{m}$ high channel structure a deformation of the channel with a dilatation of its cross-section is observed. This phenomenon induces a change of the fluidic resistance and thus a failure in the flow measurement. Figure 14 presents the results of the $25 \mu \mathrm{m}$ channel height sensor compared to theory. The pressure over the sensor has lost its linear behaviour in air. For small flow the measured pressure is higher than the calculated. The channel is constricted. With increasing flow and pressure the measuring curve rises. The soft PDMS expands from the inside by the relatively high positive pressure. The duct cross section becomes larger and flow resistance becomes smaller. In water this effect cannot be observed, since the flow like the pressures lies lower here.

Another application of the thermal flow sensor is its use as differential pressure sensor. To achieve high pressure sensitivity the internal fluidic resistance is increased by adding a meander structure to the fluid channel in PDMS on chip. An enhancement of the sensitivity by a factor of 6.3 is achieved (figure 8).

This result opens new industrial applications for the flow sensor as precise differential pressure sensor.

\section{- Adhesive-free packaging}

Finally, a concept for a biocompatible adhesivefree packaging of the sensor is presented in Figure 9. A chip containing a RIE fluid channel with a PDMS protection layer is clamped between two plastic parts. The PDMS layer protects the sensor membrane and thus increases its robustness and compensates tolerances of the packaging. Tightness is performed with two o-rings placed at the in- and out-let of the chip. The electrical connection is provided by a flexible circuit board on which the sensor chip is bonded. This package demonstrates the possibility to realize a hermetically sealed snap packaging in injection molding technology.

\section{Conclusion}

We presented a new generation of flow sensors with integrated micro-channels and demonstrated the realization of small channel structures using improved fabrication processes. The obtained sensor characteristics over different flow rates in water and air correspond with the expected improvement in sensitivity and resolution. Realizing the fluidic channels by a $\mathrm{KOH}$ etching process followed by a dry etching from the backside of the sensor element provides the 
highest geometrically reproducibility. The new sensors present a failure in reproducibility under $1 \%$ whereas the standard ones with channel integrated in the housing show about $12 \%$.

In addition to standard mass flow rate measurements the realization of a high precise differential pressure sensor was possible. Therefore the internal fluidic resistance of the flow sensor was increased by integrating different micro-channel structures on chip. This leads to a very compact system having the size of the silicon chip $\left(24 \mathrm{~mm}^{2}\right.$ and $4 \mathrm{~mm}^{2}$ with and without meander respectively). This allows the realization of very cheap housing leading to the production of low cost flow sensors in the domain of small flow range.

Using this technology an excellent reproducibility in the fluidic resistance of the flow channels over a wafer batch is obtained. A high sensitivity for the measurements of low flow rates $(0.1-5.0 \mu \mathrm{l} / \mathrm{min}$ in water) and difference pressures (10-600 $\mathrm{Pa}$ in air) is attained. It opens the doors of new applications field for the flow sensors especially in medical applications domains like air conditioning.

\section{Acknowledgments}

This research project AiF-No $14281 \mathrm{~N}$ was funded by the German Federal Ministry of Economics and Technology via grants of the German Federation of Industrial Research Associations (AiF)

\section{References}

[1] F. Völklein, A. Wiegand, V. Baier: Sensors \& Actuators A, Vol. 29, pp87-91, 1991.

[2] Gehman, Murray, Speldrich; Reduced Package Size for Medical Flow Sensor, Honeywell (presented at the IMAPS Technical Symposium), 2000.

[3] N.T. Nguyen and S.T. Wereley, Fundamentals and Applications of Microfluidics, Artech House, pp343-372, 2002

[4] Theo S. J. Lammerink, Niels R. Tas, Miko Elwenspoek and Jan H. J. Fluitman, Micro-liquid flow sensors, Sensors and Actuators A:Physical, 37-38 (1993) 45-50

[5] R.E. Oosterbroek, T.S.J. Lammerink, J.W. Berenschot, G.J.M. Krijnen, M.C. Elwenspoek and A. van den Berg, A micromachined pressure/flow-sensor Sensors and Actuators A, 77, 3 (1999) 167-177

[6] A.M. Boillant et al., A Differential Pressure liquid sensor for flow regulation and dosing systems Proceedings of MEMS'95, 8th IEEE International Workshop on Micro-electromechanical Systems" (1995) 350-352

[7] T.S. Cho and K.D.Wise, A High Performance Microflowmeter with Built-in self-test, Sensors and Actuators A, 36 (1993) 47-56

[8] L. Furuberg, D. Wang, A. Vogl, L. Solli, Flow sensor for dosing applications, Micro Structure Workshop 2004, Ystads Saltsjöbad., 2004.

[9] Mayer et al. Sensirion, United States Patent No.: 6,813,944 B2, Nov.9, 2004

[10] M. Ashauer, H. Scholz, R. Briegel, H. Sandmaier, W. Lang, Thermal Flow Sensors for very small Flow Rate; Trandsducer 2001, Munich, 4B208P, 2001

[11] K. Kliche, Diplomarbeit 2006 am HSG-IMIT Charakterisierung thermischer Strömungssensoren mit integriertem Strömungskanal, 2006. 